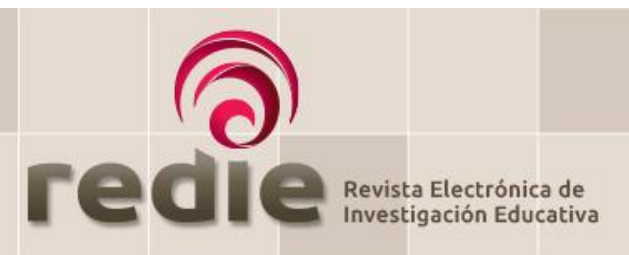

\title{
Validación de AUTOCOM: autoevaluación de las competencias básicas de jóvenes en el marco de programas formativos de segunda oportunidad
}

\author{
Validation of АUтосом: Self-Assessing Young People's Basic \\ Competencies within the Framework of Second Chance \\ Training Programs
}

Patricia Olmos Rueda (*) patricia.olmos@uab.cat

Óscar Mas Torelló (*) oscar.mas@uab.cat

(*) Universidad Autónoma de Barcelona

(Recibido: 28 de octubre de 2016; Aceptado para su publicación: 17 de abril de 2017)

Cómo citar: Olmos, P. y Mas, O. (2018). Validación de AuTocom: autoevaluación de las competencias básicas de jóvenes en el marco de programas formativos de segunda oportunidad. Revista Electrónica de Investigación Educativa, 20(4), 49-61. https://doi.org/10.24320/redie.2018.20.4.1674

\section{Resumen}

Este trabajo aborda el proceso de validación de AUTOCOM, un instrumento de autoevaluación de la percepción que los jóvenes tienen de su nivel de dominio de competencias básicas en el marco de programas formativos de segunda oportunidad y, en función de ello, considerar su contribución al proceso de autorregulación de su aprendizaje. A partir de la administración del cuestionario a una muestra piloto de 228 jóvenes, previa validación de consistencia interna (a inicial=0.91) y concordancia de expertos $(W>0 ; p<0.005)$, se realizó un análisis de conglomerados jerárquico confirmado por análisis discriminante, que identificó dos grupos de competencias básicas en consideración del nivel de dominio cluster 1: 49\% de los jóvenes; cluster 2: $51 \%$ de los jóvenes. Los resultados identifican a AuTocom como un instrumento válido y confiable en el marco de los programas formativos para evaluar, mediante la percepción de los jóvenes, el nivel de dominio de competencias básicas y su contribución al proceso de autorregulación del aprendizaje.

Palabras clave: Aprendizaje autorregulado, competencias básicas, autopercepción, abandono escolar, orientación.

\section{Abstract}

This study addresses the validation process for AUTOCOM, a self-assessment tool to evaluate young people's perceived level of proficiency in basic competencies within the framework of second-chance training programs, and on that basis considers its contribution to the self-regulation process in their learning. From a questionnaire administered to a pilot sample of 228 young people, and following validation of internal consistency (initial $a=0.91$ ) and expert agreement $(W>0 ; p<0.005)$, a hierarchical 
cluster analysis was performed and confirmed by discriminant analysis. This identified two groups of basic competencies based on the level of proficiency: cluster 1: $49 \%$ of young people; cluster $2: 51 \%$ of young people. The results show that AUTOCOM is a valid and reliable tool for evaluating, within the context of training programs, young people's perception of their level of proficiency in basic competencies and how this contributes to the self-regulation process in their learning.

Keywords: Self-regulated learning, key competencies, self-perception, academic failure, guidance.

\section{Introducción}

El abandono escolar prematuro merma el proceso de adquisición y desarrollo de competencias básicas de muchos jóvenes, competencias clave para su inclusión en contextos sociales, educativos y laborales (Pallisera, Vilà y Fullana, 2012) y los pone en riesgo de exclusión.

Las competencias básicas son aquellas competencias para el aprendizaje permanente definidas en un marco europeo de referencia -competencia comunicativa, matemática, digital, social y ciudadana, autonomía y sentido de la iniciativa, interacción con el medio y aprender a aprender; entendidas como competencias para la vida y para el desarrollo integral de la persona que permiten dar respuesta a las demandas que tanto la sociedad como un mercado de trabajo en constante evolución exigen (Comisión Europea, 2007).

El colectivo de jóvenes que abandona prematuramente sus estudios no sólo no adquiere el dominio de las competencias, tampoco obtiene el certificado educativo que le reconoce la finalización de la etapa de escolarización obligatoria, limitando su continuidad en el sistema educativo (Vallejo y Dooly, 2013). Para muchos, la alternativa son los programas formativos de segunda oportunidad, programas de formación profesional de nivel 1 de cualificación, ${ }^{1}$ que se ofertan en el contexto español y catalán, al que tienen acceso aquellos jóvenes que por abandonar prematuramente la escuela no obtuvieron el certificado de estudios mínimos obligatorios -graduado en Educación Secundaria Obligatoria- quedando excluidos de los itinerarios de educación reglados, cuyo punto de partida es la formación en competencias básicas (Olmos y Mas, 2013).

La experiencia educativa previa del perfil de jóvenes que acceden a este tipo de programas tiende a ser negativa -fracaso escolar reiterado, escasos logros académicos, falta de motivación e interés, etc.- y deriva, a menudo, en una falta de confianza en su capacidad para aprender y un bajo autoconcepto académico general.

El equipo de tutores de estos programas formativos, conscientes del perfil de los jóvenes, trabaja para que sean aprendices autorregulados; es decir, jóvenes implicados en su proceso de aprendizaje: capaces de confiar en sus capacidades para aprender (autoeficacia), que crean en el aprendizaje y quieran aprender (motivación), y que sean capaces de evaluar sus potencialidades y sus limitaciones, carencias o necesidades (autoevaluación), de acuerdo con Inglés, Martínez-Monteagudo, García-Fernández, Valle y Castejón (2015).

En el marco de los programas formativos de segunda oportunidad, los jóvenes siguen un patrón de aprendizaje de aplicación directa; es decir, aprenden en dos contextos de aprendizaje complementarios: el contexto aula (donde los jóvenes aprenden las competencias a desarrollar) y el contexto laboral (centros de trabajo donde los jóvenes realizan períodos de prácticas formativas y donde aplican las competencias aprendidas en el aula, las consolidan y adquieren mayor conciencia de sus potencialidades y limitaciones) (Martínez-Fernández y Vermunt, 2015).

\footnotetext{
${ }^{1}$ Estos programas de formación profesional responden a una clasificación cine 0-2 (Clasificación Internacional Normalizada de la Educación elaborada por la unESCO para medir los niveles de educación de los países), el nivel de clasificación educativo más bajo, y se definen como programas de formación profesional inicial o básica.
} 
Este es el marco de referencia del presente trabajo, que busca contribuir a la evaluación del nivel de dominio de las competencias básicas, a partir de la autoevaluación de los jóvenes que participan en programas formativos de segunda oportunidad desarrollados en España y más concretamente en Cataluña, por medio de la administración del instrumento AUTOCOM, cuestionario de autopercepción de competencias básicas, diseñado ex profeso para este estudio, y valorar su apoyo al proceso de autorregulación del aprendizaje.

El cuestionario permite a los jóvenes explorar y autoevaluar el nivel de dominio de las competencias básicas para el aprendizaje permanente, al identificar las carencias o necesidades que poseen y establecer metas de aprendizaje propias para la adquisición de esas competencias.

Es importante que los jóvenes entiendan la importancia que tiene la adquisición de estas competencias básicas, para ello deben ser conscientes del dominio que creen tener de las mismas. En términos de Inglés et al. (2015), el primer paso para implicar de manera activa a estos jóvenes en su proceso de aprendizaje es partir del autoconcepto, vinculado al nivel de dominio competencial e identificación de las carencias para, posteriormente, poder establecer como meta de aprendizaje la adquisición y dominio de esas competencias básicas requeridas.

Por lo tanto, este trabajo parte de la pregunta de investigación: ¿Es AUTOCOM un instrumento válido de autoevaluación en el marco de los programas formativos de segunda oportunidad para que los jóvenes puedan explorar su nivel de dominio de las competencias básicas e identificar qué necesidades presentan al respecto?

En ese escenario cabe preguntarse qué factores se vinculan a la situación de exclusión de los jóvenes. Por lo general son factores contextuales, como la influencia social y del entorno, por citar algunos, interrelacionados con factores personales, por ejemplo: autocontrol, autoconcepto, autoestima y motivación, entre otros. Côté (2005) describe estos últimos como recursos personales claves en la autoformación; es decir, recursos que capacitan al individuo para dar respuesta efectiva a los obstáculos sociales, educativos y personales que se le van presentando.

Asimismo, estos factores personales resultan ser, en opinión de Zimmerman (1990), componentes significativos de la autorregulación del aprendizaje (SRL, por sus siglas en inglés), entendidos como procesos básicos a considerar en y para los procesos de inclusión educativa y socio-laboral de los jóvenes en riesgo de exclusión; por ejemplo, porque el proceso de autorregulación les permite mejorar sus resultados de aprendizaje y su capacidad para adaptarse a contextos cambiantes (Cassidy, 2011; Zimmerman, 2002). En la idea de que lo más importante no es aquello que se sabe, sino cómo se sabe (Woolcock, 2001; Woolcock y Narayan, 2000).

El proceso de autorregulación requiere de la habilidad de seguimiento y control por parte de la persona de su proceso de aprendizaje; esto es, ser consciente de sus fortalezas, carencias o necesidades para ser proactiva en su proceso de aprendizaje (Zimmerman, 2002, 2008). Aquí es donde la autoevaluación se presenta como una de las estrategias de autorregulación clave en la medida que resulta un proceso cíclico en el que la persona que aprende debe ser consciente de lo que aprende (autocontrol), conocer sus progresos hacia las metas de aprendizaje (autojuicio) e identificar, así como implementar las medidas de corrección necesarias durante el proceso de aprendizaje, para incidir, todo ello en la autosuficiencia de la persona que aprende (McMillian y Hearn, 2008; Ross, Tronson y Siegenthaler, 2006).

De acuerdo con Zimmerman (1990), el SRL es un sistema triádico de influencias conductuales, personales y del entorno, aunque basado en la regulación personal. Por ejemplo, autores como Luyckx, Hans de Witte y Luc (2011) y Weller (2010) establecen que la persona se define a sí misma (autoconcepto) en función de sus redes sociales y de los recursos que puede adquirir de éstas. Esto es, depende de las relaciones que se establecen entre los principios de regulación de la producción social y el funcionamiento individual (Daniels, 2009). En el caso del colectivo de jóvenes con bajo rendimiento académico, a menudo son tildados como estudiantes con problemas de conducta (Jull, 2009), irresponsables, sin motivación por el aprendizaje y carentes de autocontrol. Ante esta percepción social negativa, ellos responden proyectando una imagen también negativa, especialmente en aquellos contextos en los que desarrollan 
su actividad, por ejemplo, los educativos (González-Pienda et al., 2000; Santibáñez, 2009).

Al respecto, la identidad personal se construye como miembro de estructuras sociales, de acuerdo a las percepciones de otros. Por ello, aunque pueda resultar contradictorio, el SRL no es únicamente un proceso de aprendizaje modelado por la persona, también es un proceso de aprendizaje modelado por otros, como el profesorado, los padres, y el grupo de iguales (Zimmerman, 2002; Zimmerman, Kitsantas y Campillo, 2005), pero -como se ha indicado anteriormente- supeditado a la regulación personal.

El proceso SRL implica y precisa de iniciativa personal, perseverancia y capacidad de adaptación (Zimmerman et al., 2005), tres cualidades identificadas en el marco de las denominadas competencias básicas, que contribuyen a mantener la capacidad de aprendizaje permanente-competencia de aprender a aprender-, y que son sumamente demandadas en los actuales contextos educativos, sociales y laborales. Cassidy (2011), Daura (2015), Hinojosa y Sanmartí (2016), Muñoz-San Roque, Martín-Alonso, Prieto-Navarro y Urosa-Sanz (2016) y Zimmerman (2002) aluden al significado de los procesos SRL en estos contextos y hacen referencia a la necesidad de trabajarlos por su vinculación con las competencias básicas, especialmente la competencia de aprender a aprender, entendida según Olmos (2014) como una competencia central y transversal al resto, en la medida en que esta competencia le permite a la persona tomar conciencia de su proceso de aprendizaje, ser cada vez más eficaz y autónoma y, por lo tanto, autorregular su propio proceso de aprendizaje. En otros términos, el proceso de autorregulación del aprendizaje es una variable básica y necesaria para el desarrollo de la competencia "aprender a aprender" (García-Martín, 2012; Vives-Varela, Durán-Cárdenas, Varela-Ruíz y Fortoul van der Goes, 2014).

En este marco conceptual se ubica el presente trabajo. El currículum actual del sistema educativo español de la etapa de educación obligatoria (de los 6 a los 16 años) se caracteriza por ser un currículum basado en competencias. Cuando los jóvenes finalizan sus estudios obligatorios deben haber adquirido y desarrollado un nivel de dominio suficiente de dichas competencias regidas por el marco de referencia europeo (Comisión Europea, 2007; Commission of the European Communities, 2005). Pero aquellos jóvenes que abandonan de forma prematura sus estudios no adquieren ese nivel de dominio de las competencias básicas establecidas en el currículum, viendo limitada su continuidad en el sistema educativo e incrementado su riesgo de exclusión educativa, social y laboral. Resulta, por lo tanto, evidente la necesidad de trabajar con estos jóvenes y en estrategias que permitan mejorar sus oportunidades de reinserción educativa y sociolaboral, haciéndoles protagonistas y partícipes activos de su proceso de aprendizaje.

\section{Método}

\subsection{Participantes}

Para la validación de AUTOCOM como instrumento de autoevaluación del nivel de dominio de las competencias básicas se partió de su aplicación a la muestra piloto integrada por 228 jóvenes (de 16 a 21 años de edad), participantes en 18 programas formativos de segunda oportunidad -con una media de 12 jóvenes por programa formativo- y seleccionados mediante la técnica de muestreo no probabilístico intencional por conglomerados en siete municipios de la provincia de Barcelona -Hospitalet de Llobregat, Sant Feliu de Llobregat, Vilanova i la Geltrú, Sabadell, Montcada y Reixach, Granollers y Pineda de Mar. La característica común de los jóvenes de la muestra fue su condición sine qua non de acceso a los programas formativos: no estar en posesión del certificado de estudios obligatorios, por lo que hablamos de jóvenes con una baja cualificación y un perfil educativo marcado por el fracaso y el abandono escolar prematuro; es decir, con un bajo nivel de dominio de competencias básicas. La aplicación de la prueba chi-cuadrado de homogeneidad de la distribución de frecuencias no identifica diferencias estadísticamente significativas entre los grupos de edad por municipio $\left(x^{2}=12.2 ; p=.057\right)$.

\subsection{Instrumento}

AUtocom es un cuestionario diseñado ex profeso para este estudio con el propósito de que sea una herramienta de evaluación del nivel de dominio de competencias básicas en los jóvenes participantes en 
los programas formativos de segunda oportunidad. Para la confección del instrumento se tomaron en cuenta aspectos vinculados al perfil de los jóvenes a los que iba dirigido (jóvenes entre 16 y 21 años con un bajo nivel formativo), el tiempo de respuesta del cuestionario (se procuró que fuera lo más breve posible, máximo 30 minutos), la redacción de los ítems (clara, precisa, unívoca y directa, que no provocaran hostilidad o rechazo en la respuesta), la variable de estudio (las competencias básicas) y la fundamentación teórica. Con relación a este último aspecto, los ítems de AUTOcom se formularon a partir de la revisión de otros instrumentos existentes en el campo de investigación sobre autoconcepto: Cuestionario multidimensional de autoconcepto (García-Gómez, 2001); Self Description Questionnaire (SQD) (Elexpuru, 1992; Marsh, 1990); Escala Sydney Attribution Scale (SAS) (Inglés, Rodríguez-Marín y González-Pienda, 2008; Marsh, 1984); Escala de autoestima y autoconcepto (Rosenberg, 1973); prueba de autoestima (clasificación Q) adaptada al contexto español (Villa, 1992), y de la concepción de un modelo integral y combinado de competencias básicas para la empleabilidad (Olmos, 2014). Dicho modelo, basado en Schalock (2009), el proyecto DeSeCo de la OCDE (2009) y la propuesta de recomendación sobre competencias clave (Commission of the European Communities, 2005), identifica dimensiones claves para el funcionamiento humano: habilidad intelectual, comportamiento adaptativo, participación social, interacción en el contexto, salud y la dimensión transversal aprender a aprender.

Para el correcto funcionamiento de cada una de estas dimensiones se precisa la adquisición y desarrollo de unas competencias básicas que AUTOCom identifica a través de 50 ítems, como se detalla a continuación: la dimensión habilidad intelectual identifica las competencias: comunicativa (6 ítems que exploran la comprensión lectora y la expresión oral y escrita); matemática (4 ítems que exploran el razonamiento lógico, espacial y abstracto) y la digital (4 ítems que exploran la habilidad digital). La dimensión comportamiento adaptativo identifica la competencia de autonomía e iniciativa personal (5 ítems que exploran la actuación autónoma, la iniciativa y pensar la capacidad emprendedora); la dimensión salud identifica la competencia salud (10 ítems que exploran hábitos de bienestar físico y emocional); la dimensión participación social identifica la competencia socio-laboral y ciudadana (con 12 ítems que exploran la capacidad de interacción, adaptación, disponibilidad, polivalencia o conocimiento del mercado de trabajo); la dimensión interacción con el contexto identifica la competencia de interacción con el medio (4 ítems que exploran la capacidad naturalista y ecológica) y, por último, la dimensión transversal que identifica la competencia de aprender a aprender (5 ítems que exploran la actitud hacia el aprendizaje).

Cada uno de los ítems formulados hace referencia a situaciones o actividades cotidianas relacionadas con las competencias básicas evaluadas y con las que los jóvenes están familiarizados. Asimismo, cada uno de los ítems requiere, por parte de los jóvenes, una respuesta valorativa de la situación que se les plantea según una escala 1 a 4 (donde 1=No, nunca; 2=Más bien pocas veces, 3=Algunas veces, 4=Sí, siempre). ${ }^{2}$

\subsection{Procedimiento y análisis de los datos del proceso de validación de AUTOCOM}

Para la validación del cuestionario AUTOCOM como herramienta de autoevaluación del nivel de dominio de competencias básicas se realizó un doble proceso de validación. En una primera fase, previa a la aplicación del cuestionario, se validó el instrumento (cuestionario) mediante juicio de expertos. Un total de 11 expertos validaron la pertinencia (1=Sí / 2=No; univocidad 1=Sí / 2=No e importancia escala 1-5, siendo 1 el valor mínimo y 5 el valor máximo) de los 55 ítems iniciales del cuestionario, que posterior a la validación por jueces, se redujeron a 50. Para valorar el nivel de concordancia entre los expertos, los resultados se sometieron a la prueba estadística coeficiente de concordancia W de Kendall. En una segunda fase de validación, los resultados se sometieron a las pruebas estadísticas de consistencia interna (alfa de Cronbach) y de concordancia (coeficiente de concordancia W de Kendall). En una primera etapa, ambas pruebas se aplicaron a las puntuaciones directas de cada uno de los ítems y, en una segunda etapa, se

\footnotetext{
${ }^{2}$ A modo de ejemplo se exponen algunas de la situaciones planteadas por los ítems para la identificación del nivel de dominio de las competencias básicas: Competencia digital (Chateo diariamente con mis amigos/as: Messenger, Skype, Gmail); Competencia comunicativa (Me resulta fácil hablar con otra persona; Entiendo lo que leo); Competencia matemática (Se me da bien resolver problemas matemáticos); Competencia aprender a aprender (Cuando un tema me interesa busco más información).
} 
aplicaron a las puntuaciones derivadas obtenidas después de haber estandarizado las puntuaciones directas de cada uno de los ítems, de acuerdo a las competencias básicas exploradas con este instrumento.

Después de pilotearse el instrumento, con el propósito de explorar qué posibles grupos de jóvenes similares y homogéneos podían identificarse en el marco de estos programas formativos respecto a su percepción del nivel de dominio de las competencias básicas evaluadas por medio de AUTOCOM, se aplicó el análisis estadístico de conglomerados a las puntuaciones estandarizadas. El primer análisis aplicado a los datos responde al método jerárquico y aglomerativo para deducir los posibles grupos (Vilà-Baños, Rubio-Hurtado, Berlanga-Silvente y Torrado-Fonseca, 2014). Un segundo análisis responde al análisis Kmedias estableciendo como número de conglomerados los obtenidos del primer análisis y confirmar así la formación de los grupos homogéneos de jóvenes en función de la percepción del nivel de dominio de las competencias básicas autoevaluadas.

Finalmente, con el propósito de confirmar y completar la formación de los grupos resultantes, se hizo un análisis discriminante para verificar la existencia de relaciones causales entre la pertenencia a un grupo u otro y los valores que adoptan las variables.

Todos los análisis estadísticos fueron realizados con el programa estadístico spss versión 17.0 y, producto de estos análisis estadísticos, se anticipa la identificación de AUTOCOM como instrumento válido, consistente y confiable para la evaluación del nivel de dominio de competencias básicas por parte de los jóvenes y el establecimiento de grupos de jóvenes con perfiles similares y homogéneos en función de la percepción que poseen de su nivel de dominio de las competencias básicas que se trabajan en el marco de los programas formativos de segunda oportunidad, tal y como se presenta a continuación.

\section{Resultados}

En cuanto a la evaluación mediante juicio de expertos, los resultados establecen un valor estadístico $W$ superior a 0 ( $W=0.246$ para pertinencia; $W=0.436$ para univocidad; $W=0.478$ para importancia) y el valor del nivel crítico es inferior a 0.005 ( $p=0.000$ para todas las variables o criterios de validación), por lo que se acepta la hipótesis de concordancia y se concluye que existe asociación significativa entre las puntuaciones de las variables analizadas por parte de los expertos respecto de los ítems del cuestionario. El $87.3 \%$ de los jueces valoraron de forma positiva la pertinencia de los ítems, el 85.5\% su univocidad y el valor medio de la importancia fue de 4.2 (sd=0.32) en una escala 1-5.

El análisis de la consistencia interna y concordancia de los ítems (puntuaciones directas) ofrece un valor alfa de Cronbach bueno $(a=0.91)$ y un valor estadístico $W$ superior a 0 con significación estadística $(W=0.192 ; p=0.000<0.005)$ lo que permite confirmar la fiabilidad del cuestionario, así como el nivel de concordancia entre las respuestas dadas por los jóvenes a los ítems.

Los resultados de consistencia y concordancia del cuestionario, después de haber estandarizado las puntuaciones directas de cada uno de los ítems de acuerdo c las competencias básicas exploradas, un total de 8 , establecen un valor alfa de Cronbach significativo ( $a=0.74$ ) que confirma la coherencia interna del cuestionario y un valor estadístico $W$ superior a 0 con significación estadística ( $W=0.217$; $p=0.000<0.005)$, el cual sigue indicando que se mantiene el nivel de concordancia entre las respuestas dadas por los jóvenes con relación a cada una de las competencias analizadas.

Es interesante apuntar que los rangos medios de respuesta identifican sus valores más bajos en las competencias con carácter más instrumental (3.39 para las competencias comunicativa y matemática y 2.87 para la competencia interacción con el medio), lo que podría estar vinculado a la relación que los jóvenes participantes de la muestra establecen entre estas competencias y su experiencia académica previa que anticipa una posible agrupación.

El procedimiento seguido para determinar qué posibles grupos similares y homogéneos de jóvenes pueden establecerse en el marco de los programas formativos de este trabajo, en función de la 
percepción que estos tienen de su nivel de dominio de competencias básicas, responde a un análisis de conglomerado jerárquico que: 1) conglomera las variables de análisis (las 8 competencias básicas que explora el cuestionario); 2) establece el historial de conglomeración que muestran las variables combinadas en cada etapa, la distancia entre las variables y el último nivel de proceso en el que cada variable se unió a su conglomerado; 3) representa gráficamente los pasos del conglomerado jerárquico mediante el dendograma, utilizando una vinculación de Ward y un diagrama de dispersión de los grupos identificados con relación al perfil de jóvenes en el marco de los programas formativos de este trabajo, en función de la percepción que tienen del nivel de dominio de las competencias básicas evaluadas.

Los resultados establecen dos conglomerados (clusters) que se combinan en 7 etapas de análisis: a) Cluster 1, integrado por las competencias salud, socio-laboral y ciudadana, aprender a aprender, autonomía e iniciativa y digital, caracterizadas por una percepción alta de su nivel de dominio; b) Cluster 2, integrado por las competencias comunicativa, matemática e interacción con el medio, caracterizadas por una percepción baja de su nivel de dominio (ver tabla I).

Tabla I. Historial de conglomeración

\begin{tabular}{c|c|c|c|c|c|c}
\hline \multirow{2}{*}{ Etapa } & \multicolumn{2}{|c|}{$\begin{array}{c}\text { Conglomerados } \\
\text { que se combinan }\end{array}$} & \multirow{2}{*}{ coeficientes } & \multicolumn{2}{|c|}{$\begin{array}{c}\text { Etapa en la que aparece } \\
\text { el conglomerado }\end{array}$} & \multirow{2}{*}{$\begin{array}{l}\text { Próxima } \\
\text { etapa }\end{array}$} \\
\cline { 2 - 3 } & Congl. 1 & Congl. 2 & & 0 Congl. 1 & Congl. 2 & \\
\hline 1 & 5 & 7 & 16.265 & 0 & 0 & 2 \\
2 & 5 & 8 & 45.514 & 1 & 0 & 4 \\
3 & 1 & 2 & 79.421 & 0 & 0 & 6 \\
4 & 4 & 5 & 116.958 & 0 & 2 & 5 \\
5 & 3 & 4 & 183.411 & 0 & 4 & 7 \\
6 & 1 & 6 & 270.224 & 3 & 0 & 7 \\
7 & 1 & 3 & 421.927 & 6 & 5 & 0 \\
\hline
\end{tabular}

Para confirmar la formación de los dos grupos obtenidos (grupo 1, integrado por los jóvenes que perciben un alto nivel de dominio de las competencias básicas salud, sociolaboral y ciudadana, aprender a aprender, autonomía e iniciativa y digital, y grupo 2, integrado por los jóvenes que perciben un bajo nivel de dominio de las competencias básicas comunicativa, matemática e interacción con el medio -e interpretarlos en el conjunto de los jóvenes de la muestra; es decir, determinar si estos jóvenes pueden integrar y distribuirse en ambos grupos, se aplica un segundo análisis de conglomerado no jerárquico (Kmedias) para el que se determinaron los dos conglomerados obtenidos del primer análisis y se observó que existía convergencia de la solución antes de las 10 iteraciones.

Los resultados muestran que el modelo de dos grupos cumple con el criterio de convergencia antes de las 7 iteraciones, y que los conglomerados finales caracterizan dos perfiles de jóvenes, respecto a la autoevaluación del nivel de dominio de las competencias, con base en su percepción: un primer perfil (cluster 1), integrado por 112 jóvenes con una percepción moderada de su nivel de dominio de las competencias, destacando la valoración desfavorable que hacen de las competencias más instrumentales; un segundo perfil (cluster 2), integrado por 115 jóvenes que, en general, poseen una percepción favorable de su nivel de dominio de las competencias aunque, como pasa con el primer grupo, la percepción de las competencias más instrumentales también tiende a ser inferior.

Finalmente, para completar y confirmar la formación de estos dos grupos se hizo un análisis discriminante. El procedimiento seguido fue: 1) contrastar la igualdad de las matrices de covarianza de los grupos aplicando el test $M$ de Box (prueba paramétrica discriminante que minimiza la probabilidad de equivocarse al clasificar los sujetos en los dos grupos identificados); 2) estimar la variabilidad inter-grupo explicada en la función discriminante y qué varianza total de las puntuaciones discriminantes no ha sido explicada por la diferencia entre grupos (autovalor y lambda de Wilks respectivamente); 3 ) determinar si existen diferencias de comportamiento entre las medias mediante el test de contraste de la lambda de Wilks; 4) identificar qué variables (competencias) son más influyentes en las funciones discriminantes y el modelo predictivo mediante la tabla de coeficientes estandarizados; 5) clasificar a los jóvenes en cada uno de los grupos a partir de la aplicación de la función discriminante obtenida. 
Los resultados del test $M$ de Box $(p=0.000<0.005)$ confirman que las matrices de varianza-covarianza son diferentes y permiten concluir que uno de los grupos es más variable que el otro. Como el autovalor obtenido se aleja de 0 (2.095), la correlación canónica resultante es alta (0.823) y el valor lambda de Wilks es próximo a $0\left(\lambda=.323\right.$; siendo su valor transformado $\left.x^{2}=250.82 ; p=0.000\right)$, de esta manera, se puede concluir que las variables discriminantes utilizadas permiten, de forma significativa, identificar a los jóvenes en uno u otro grupo e interpretar, por una parte, que los grupos son diferentes y, por otra, que la función discriminante puede pronosticar adecuadamente a los jóvenes en cada uno de ellos.

Asimismo, el contraste de lambda de Wilks $(p=0.000)$ establece diferencias de comportamiento entre las medias de los dos grupos identificados y la tabla de coeficientes estandarizados identifica qué competencias son las más influyentes, quedando excluidas la competencia socio-laboral y ciudadana y la competencia autonomía-iniciativa.

Por último, la aplicación de la función discriminante permite clasificar correctamente al $97.8 \%$ de los jóvenes participantes en cada uno de los grupos. En el primero, a aquellos jóvenes que tienen una percepción desfavorable de su nivel de dominio de competencias básicas y no suelen mejorar esa autopercepción; en el segundo, a aquellos jóvenes que tienen una percepción favorable de su nivel de dominio de las competencias básicas y, ocasionalmente, pueden tender a una autopercepción más negativa, conformando el grupo más variable y confirmando los resultados previamente obtenidos en los análisis de conglomerados previos.

\section{Discusión y conclusiones}

El principal propósito de este trabajo era determinar si AuTOcom resulta un instrumento de autoevaluación válido en el marco de los programas de segunda oportunidad para que los jóvenes exploren su nivel de dominio de competencias básicas, identifiquen qué necesidades presentan al respecto y, de esta manera, contribuir al proceso de autorregulación de su aprendizaje. Sometido AUTOCOM a proceso de validación, se observa que el cuestionario es un instrumento válido a tal fin y, en la línea de otros estudios e investigaciones (Bourke y Mentis, 2013; García-Martín, 2012; Gómez, Rodríguez e Ibarra, 2013; Kostons, Van Gog y Paas, 2012; Rodríguez-Mena y García, 2001; Rodríguez-Mena, García, Corral y Lago, 2004; Vives-Varela et al., 2014), un recurso que contribuye al fortalecimiento de la identidad de los jóvenes como aprendices competentes, estratégicos y autorregulados, si partimos de la premisa de que todo aprendiz competente y autorregulado debe conocer, evaluar y reflexionar sobre sus capacidades y conocimientos (en el marco de este trabajo entiéndase eso como competencias básicas) y ser sujeto activo y participativo de su proceso de aprendizaje (Monereo, 2001; Torre, 2007).

Tal y como se apunta, todo proceso de autorregulación requiere que un aprendiz autorregulado sea capaz de activar las competencias que contribuyen a su proceso de aprendizaje y ello será posible si es capaz de conocerse a sí mismo (García-Martín, 2012; Monereo, 2001; Muñoz-San Roque et al., 2016; Zimmerman, 2013). En el marco de este trabajo y de los programas formativos considerados en el mismo (programas formativos de segunda oportunidad), es preciso partir del autoconcepto y autopercepción de las potencialidades y necesidades de los jóvenes en relación con el proceso de aprendizaje, focalizado en la adquisición y desarrollo de ocho competencias básicas. Así, AUTOCOM contribuye a la exploración, por parte de los jóvenes, de su nivel de dominio de competencias básicas, estrechamente vinculadas a lo que otras líneas de investigación han establecido como dimensiones de autoconcepto (Inglés et al., 2015), y permite a los jóvenes ser actores activos y participativos de su proceso de aprendizaje.

Por otro lado, los resultados también confirman a AUTOCOM como un instrumento que contribuye al establecimiento de las competencias básicas como variables discriminantes que clasifican a los jóvenes en dos grupos en relación con la percepción que tienen de su nivel de dominio del conjunto de las competencias analizadas, aspecto también vinculado a los procesos de autorregulación del aprendizaje. En este sentido, se encontró un grupo de jóvenes que se caracteriza por tener una percepción favorable de su nivel de dominio de competencias, especialmente no instrumentales (por ejemplo, autonomía e iniciativa personal o competencia socio-ciudadana y laboral) y, por otra, el grupo de jóvenes con una percepción negativa respecto al nivel de dominio de competencias, especialmente instrumentales 
(comunicativa o matemática, por ejemplo). De acuerdo con Gómez, Rodríguez e Ibarra (2013), esta sobreestimación o subestimación de los niveles competenciales autopercibidos puede resultar un elemento positivo y favorecedor de los procesos de autorregulación del aprendizaje en la medida que contribuyen a un mejor autoconcepto y confianza de los jóvenes en sus potencialidades de aprendizaje y oportunidades formativas (en el caso de la sobreestimación), o a ser conscientes de la necesidad de tener que mejorar su formación (en el caso de la subestimación). De esta manera, AUTocom también puede ser considerado un instrumento o recurso de retroalimentación positiva para los jóvenes respecto a su proceso de aprendizaje.

Cabe resaltar que en el marco referencial de este trabajo, a pesar de las diferencias entre ambos grupos de jóvenes, se observan tendencias de autopercepción similares, vinculadas especialmente con las competencias de carácter más instrumental. Son estas competencias las que los jóvenes de ambos grupos autoperciben como menos favorables. Sobre esto, estudios como los de Dooley y Prause (1997); Hair, Moore, Ling, Mcphee-Baker y Brown (2009); Mcwhirter y Mcwhirter (2008); Martínez, Mendizábal y Sostoa (2009) y Olmos (2014) establecen que lo primero que los jóvenes mejoran al acceder a programas formativos de segunda oportunidad es la autopercepción que tienen de sus capacidades personales, que se asocian a las competencias no instrumentales, pero no ocurre lo mismo con las competencias más instrumentales, en las que perciben un nivel de competencia menos favorable. Ello puede llevar a hipotetizar que las competencias más instrumentales son las que los jóvenes vinculan directamente a contextos más académicos, en los que han fracasado reiteradamente y han conseguido escasos logros académicos. Sería interesante estudiar esta relación en futuros estudios.

Quizá una de las limitaciones de este trabajo respecto a la contribución de AUTOcom a los procesos de autorregulación del aprendizaje, ha sido el no considerar otras variables discriminantes, interrelacionadas e integrantes de los citados procesos, como las referida por García-Martín (2012), Monereo (2007) y Pintrich (2003), tales como variables personales (edad, condición de inmigrante, situación socioeconómica, estructura familiar, etc.), motivacionales (control sobre los resultados, capacidad para afrontar una tarea, expectativas, etc.), cognitivas (activación de conocimientos previos o contextuales, por ejemplo experiencia académica previa, experiencia laboral, etc.) Aunque establecer las competencias básicas resulta interesante en el marco del aprendizaje autorregulado, para próximos estudios se sugiere analizar qué otras variables personales, motivacionales, cognitivas o contextuales influyen en la discriminación de los grupos, con el objetivo de entender mejor por qué la autopercepción de algunos de estos jóvenes es más favorable que la de otros y, de esta manera, contribuir a la mejora del cuestionario como instrumento de exploración y como recurso que contribuye a la autorregulación del aprendizaje.

Por lo tanto, con el objetivo de determinar si el cuestionario AUTOCom es un instrumento válido de autoevaluación de la percepción e identificación de las necesidades formativas que tienen, se concluye que -a pesar de las limitaciones apuntadas- los resultados de este trabajo confirman que es posible establecer a AUTOCOM como un instrumento válido para tal fin, en la medida que permite a los jóvenes implicarse y participar activamente en su proceso de aprendizaje mediante la exploración de su nivel de dominio de competencias básicas (eje curricular de los actuales contextos educativos y claves para la mejora de los procesos de inserción educativa y socio-laboral) vinculadas a los procesos de aprendizaje a lo largo de la vida, contribuyendo, de esta manera, al cambio cualitativo al que Gutiérrez-Вraojos, Salmerón-Pérez y Muñoz-Cantero (2014) hacen referencia cuando los aprendices pasan de estar regulados externamente a estarlo internamente.

\section{Referencias}

Bourke, R. y Mentis, M. (2013). Self-assessment as a process for inclusion. International Journal of Inclusive Education, 17(8), 854-867. doi:10.1080/13603116.2011.602288

Cassidy, S. (2011). Self-regulated learning in higher education: identifying key component processes. Studies in Higher Education, 36(8), 989-1000. doi: 10.1080/03075079.2010.503269 
Comisión Europea (2007). Competencias clave para el aprendizaje permanente un marco de referencia europeo. Luxemburgo: Oficina de Publicaciones Oficiales de las Comunidades Europeas.

Commission of the European Communities (2005). Proposal for a Recommendation of the European Parliament and of the Council on key competences for lifelong learning. Recuperado de http://lifelonglearning-observatory.eucen.eu/recommendationcompetences

Côté, J. E. (2005). Identity capital, social capital and the wider benefits of learning: generating resources facilitative of social cohesion. London Review of Education, 3(3), 221-237.

doi:10.1080/14748460500372382

Daniels, H. (2009). Situating pedagogy: moving beyond an interactional account. Pedagogies: An International Journal, 5(1), 27-36. doi:10.1080/15544800903406282

Daura, F. T. (2015). Aprendizaje autorregulado y rendimiento académico en estudiantes del ciclo clínico de la carrera de Medicina. Revista Electrónica de Investigación Educativa, 17(3), 28-45. Recuperado de https://redie.uabc.mx/redie/article/view/408

Dooley, D. y Prause, J. (1997). Effect of students' self-esteem on later employment status: interactions of self-esteem with gender and race. Applied Psychology: An International Review, 46(2), 175-198. doi:10.1111/j.1464-0597.1997.tb01223.x

Elexpuru, I. (1992). Cinco estudios empíricos: el autoconcepto en los alumnos de 8 a 11 años de edad a través del SDQ. En A. Villa (Coord.), Autoconcepto y educación: teoría, medida y práctica pedagógica (pp. 123189). Vitoria-Gasteiz: Servicio Central de Publicaciones del Gobierno Vasco.

García-Gómez, A. (2001). Desarrollo y validación de un cuestionario multidimensional de autoconcepto. Revista Iberoamericana de Diagnóstico y Evaluación Psicológica, 11(1), 29-54. Recuperado de http://www.aidep.org/es/node/173

García-Martín, M. (2012). La autorregulación académica como variable explicativa de los procesos de aprendizaje universitario. Profesorado. Revista de Currículum y Formación del Profesorado, 16(1), 203-221. Recuperado de https://www.ugr.es/ recfpro/rev161ART12.pdf

Gómez, M. A., Rodríguez, G. e Ibarra, M. S. (2013). compes: Autoinforme sobre las competencias básicas relacionadas con la evaluación de los estudiantes universitarios. Estudios sobre Educación, 24, 197-224.

González-Pienda, J. A., Núñez, J. C., González-Pumariega, S., Álvarez, L., Roces, C., García, M., González, P., Cabanach, R. G. y Valle, A. (2000). Autoconcepto, proceso de atribución causal y metas académicas en niños con y sin dificultades de aprendizaje. Psicothema, 12(4), 548-556.

Gutiérrez-Braojos, C., Salmerón-Pérez, H. y Muñoz-Cantero, J. M. (2014). El efecto modulador de los patrones temporales sobre el logro en el aprendizaje autorregulado. Revista de Psicodidáctica, 19(2), 267287.

Hair, E. C., Moore, K. A., Ling, T. J., Mcphee-Baker C. y Brown, B. (2009). Youth who are "disconnected" and those who then reconnect: assessing the influence of family, programs, peers and communities. Child Trends, 37, 1-8. Recuperado de http://www.childtrends.org/wp-content/uploads/2013/04/8.pdf

Hinojosa, J. y Sanmartí, N. (2016). Promoviendo la autorregulación en la resolución de problemas de física. Ciênc. Educ., Bauru, 22(1), 7-22. doi:10.1590/1516-731320160010002

Inglés, C. J., Martínez-Monteagudo, M. C., García-Fernández, J. M., Valle, A. y Castejón, J. L. (2015). Perfiles de orientaciones de metas y autoconcepto de estudiantes de Educación Secundaria. Revista de Psicodidáctica, 20(1), 99-116. doi:10.1387/RevPsicodidact.1023 
Inglés, C. J., Rodríguez-Marín, J. y González-Pienda, A. (2008). Adaptación de la Sydney Attribution Scale en población universitaria española. Psicothema, 20(1), 166-173.

Jull, S. K. (2009). Student behaviour self-monitoring enabling inclusion. International Journal of Inclusive Education, 13(5), 489-500. doi:10.1080/13603110701841315

Kostons, D., Van Gog, T. y Paas, F. (2012). Training self-assessment and task-selection skills: a cognitive approach to improving self-regulated learning. Learning and Instruction, 22(2), 121-132.

doi:10.1016/j.learninstruc.2011.08.004

Luyckx, K. y Hans De Witte, H. y Luc, G. (2011). Perceived instability in emerging adulthood: The protective role of identity capital. Journal of Applied Developmental Psychology, 32(3), 137-145.

doi: 10.1016/j.appdev.2011.02.002

Marsh, H. W. (1984). Relations among dimension of self-attribution, dimension of self-concept and academic achievements. Journal of Educational Psychology, 76(6), 1291-1308.

doi:10.1037/0022-0663.76.6.1291

Marsh, H. W. (1990). The structure of academic self-concept: the Marsh/Shavelson Model. Journal of Educational Psychology, 82(4), 623-636.

Martínez, B., Mendizábal, A. y Sostoa, V. P. (2009). Una oportunidad para que jóvenes que fracasan en la escuela puedan salir de la zona de riesgo de exclusión. La experiencia de los centros de iniciación profesional en la comunidad autónoma vasca. Profesorado. Revista de Currículum y Formación de Profesorado, 13(3), 239-271.

Martínez-Fernández, R. y Vermunt, J. D. (2015). A cross-cultural analysis of the patterns of learning and academic performance of Spanish and Latin-American undergraduates. Studies in Higher Education, 40(2), 278-295. doi:10.1080/03075079.2013.823934

McMillian, J. y Hearn, J. (2008). Student self-assessment: the key to stronger student motivation and higher achievement. Educational Horizon, 87(1), 40-49.

Mcwhirter, E. H. y Mcwhirter, B. T. (2008). Adolescent future expectations of work, education, family, and community. Development of a new measure. Youth y Society, 40(2), 182-202.

doi: 10.1177/0044118X08314257

Monereo, C. (2001). Enseñar a aprender, una vieja aspiración con nuevas coordenadas. Escola Catalana, 376, 6-9.

Monereo, C. (2007). Hacia un nuevo paradigma del aprendizaje estratégico: el papel de la mediación social, del self y de las emociones. Revista Electrónica de Investigación Psicoeducativa, 5(3), 497-534.

Muñoz-San Roque, I., Martín-Alonso, J. F., Prieto-Navarro, L. y Urosa-Sanz, B. (2016). Autopercepción del nivel de desarrollo de la competencia de aprender a aprender en el contexto universitario: propuesta de un instrumento de evaluación. Revista de Investigación Educativa, 34(2), 369-383.

doi:10.6018/rie.34.2.235881

OCDE (2009). La definición y selección de competencias clave. Resumen ejecutivo. Recuperado de http://files.sld.cu/bmn/files/2014/07/la-definicion-de-competencias-claves.pdf

Olmos, P. y Mas, O. (2013). Youth, academic failure and second chance training programmes. Revista Española de Orientación y Psicopedagogía, 24(1), 78-93. Recuperado de

http://revistas.uned.es/index.php/reop/article/view/11272 
Olmos, P. (2014). Competencias básicas y procesos perceptivos: factores claves en la formación y orientación de los jóvenes en riesgo de exclusión educativa y sociolaboral. Revista de Investigación Educativa, 32(2), 531-546. doi:10.6018/rie.32.2.181551

Pallisera, M., Vilà, M. y Fullana, J. (2012). Beyond school inclusion: secondary school and preparing for labour market inclusion for young people with disabilities in Spain. International Journal of Inclusive Education, 16(11), 1115-1129. doi:10.1080/13603116.2010.548104

Pintrich, P. R. (2003). A motivational science perspective on the role of student motivation in learning and teaching contexts. Journal of Educational Psychology, 95(4), 667-686. doi:10.1037/0022-0663.95.4.667

Rodríguez-Mena, M. y García, I. (2001). Aprendizaje para el cambio. Proyecto para la Formación de aprendices autorregulados en Comunidades de Aprendizaje. La Habana: CIPS.

Rodríguez-Mena, M., García, I., Corral, R. y Lago, C. (2004). Aprender en la Empresa. Fundamentos sociopsicopedagógicos del Programa para la Formación de aprendices autorregulados en Comunidades de Aprendizaje. La Habana: Prensa Latina.

Rosenberg, M. (1973). La autoimagen del adolescente y la sociedad. Buenos Aires: Paidós.

Ross, P., Tronson, D. y Siegenthaler, S. (2006). Assessment for learning and motivation. Proceedings of The Australian Conference on Science and Mathematics Education (formerly UniServe Science Conference). Recuperado de http://openjournals.library.usyd.edu.au/index.php/IISME/article/view/6406/7044

Santibáñez, J. (2009). Diferencias entre estima: del alumno hacia sí mismo, percibida desde los demás y dirigida hacia los otros. Educar, 44, 97-119.

Schalock, R. (2009). La nueva definición de discapacidad intelectual, los apoyos individuales y los resultados personales. En, M. A. Verdugo, T. Nieto, B. Jordán de Urríes y M. Crespo, (Coords.), Mejorando resultados personales para una vida de calidad (pp. 69-88). Salamanca: Amarú.

Torre, J. C. (2007). Una triple alianza para un aprendizaje universitario de calidad. Madrid: Universidad Pontificia Comillas de Madrid.

Vallejo, C. y Dooly, M. (2013). Early school leavers and social disadvantage in Spain: from books to bricks and vice-versa. European Journal of Education, 48(3), 390-404. doi:10.1111/ejed.12037

Vilà-Baños, R., Rubio-Hurtado, M. J., Berlanga-Silvente, V. y Torrado-Fonseca, M. (2014). Cómo aplicar un cluster jerárquico en SPSS. Revista d'Innovació i Recerca en Educació, 7(1). doi:10.1344/reire2014.7.1716

Villa, A. (Coord.) (1992). Autoconcepto y educación. Teoría, medida y práctica pedagógica. Colección: Estudios y Documentos número 17. Vitoria-Gasteiz: Servicio Central de Publicaciones del Gobierno Vasco.

Vives-Varela, T., Durán-Cárdenas, C., Varela-Ruíz, M. y Fortoul van der Goes, T. (2014). La autorregulación en el aprendizaje, la luz de un faro en el mar. Investigación en Educación Médica, 3(9), 34-39.

Weller, S. (2010). Young people's social capital: complex identities, dynamic networks. Ethnic and Racial Studies, 33(5), 872-888. doi:10.1080/01419870903254653

Woolcock, M. (2001). The place of social capital in understanding social and economic outcomes. Isuma Canadian Journal of Policy Research, 2(1), 11-17.

Woolcock, M. y Narayan, D. (2000). Social capital: implications for development theory, research and policy. World Bank Research Observer, 15(2), 225-250. doi:10.1093/wbro/15.2.225 
Zimmerman, J. B. (1990). Self-regulated learning and academic achievement: an overview. Educational Psychologist, 25(1), 3-17.

Zimmerman, J. B. (2002). Becoming a self-regulated learner: an overview. Theory into practice, 41(2), 6470. doi:10.1207/s15430421tip4102_2

Zimmerman, J. B. (2008). Investigating self-regulation and motivation: historical background, methodological developments, and future prospects. American Educational Research Journal, 45(1), 166183. doi: $10.3102 / 0002831207312909$

Zimmerman, J. B., Kitsantas, A., y Campillo, M. (2005). Evaluación de la autoeficacia regulatoria: una perspectiva social cognitiva. Revista Evaluar, 5. Recuperado de

https://revistas.unc.edu.ar/index.php/revaluar/article/view/537/477

Zimmerman, J. B. (2013). From cognitive modeling to self-regulation: a social cognitive career path. Educational Psychologist, 48(3), 135-147. doi:10.1080/00461520.2013.794676 